\title{
PROGRAMA SOCIOAMBIENTAL E OS PRINCÍPIOS DE RELACIONAMENTO COM OS STAKEHOLDERS
}

\section{SOCIO-ENVIRONMENTAL PROGRAM AND THE PRINCIPLES OF RELATIONSHIP WITH STAKEHOLDERS}

\author{
Adelaide Maria Bogo*E-mail:adelaide.bogo@udesc.br \\ Elisa Henning** E-mail: elisa.henning@udesc.br \\ Universidade do Estado de Santa Catarina (UDESC), Joinville, SC, Brasil
}

\begin{abstract}
Resumo: Atualmente é comum as organizações demonstrarem um comportamento socialmente responsável e pautado nas relações com os stakeholders. Essa relação, por sua vez, baseia-se em dois princípios de relacionamento, a Legitimidade Corporativa e Stakeholder Fiduciário. Esses dois princípios trazem em sua essência o dever dos gestores de gerir a empresa de forma a atender aos stakeholders. A partir dessa percepção, a Termotécnica, uma empresa que usa poliestireno expandido (EPS) em seu processo produtivo, desenvolveu o Programa Reciclar EPS. Portanto, com base nessas informações este estudo teve como objetivo demonstrar como um programa socioambiental de reciclagem contribui para atender aqueles dois princípios. Este é um estudo de caso único, apoiado na proposição teórica de que programas socioambientais atendem aos princípios de relacionamento com os stakeholders e adota a técnica da Análise de Conteúdo e sistema Nvivo para auxiliar na organização e análise dos dados. Os resultados indicam que o Programa Reciclar EPS proporciona aos gestores legitimidade corporativa e assegura a confiança nas relações fiduciárias, portanto, a licença social para operar.
\end{abstract}

Palavras-chave: Socioambiental. Legitimidade. Fiduciário. Stakeholder.

Abstract: It is common nowadays for organizations to demonstrate socially responsible behavior based on relationships with stakeholders. These relationships, in turn, are based on two relationship principles, Corporate Legitimacy and Fiduciary Stakeholder. At their core, these two principles lead to the duty of managers to manage the company in order to serve the stakeholders. Based on this view, Termotécnica, a company that uses expanded polystyrene (EPS) in its production process, has developed the Recycle EPS program. Based on this information, this study will demonstrate how a socio-environmental recycling program contributes to these two principles. This is a unique case study, supported by the theoretical proposition that socio-environmental programs meet the stakeholder relationship principles. It adopts the content analysis technique and the Nvivo system to assist in the organization and analysis of data. The results indicate that the Recycle EPS program provides managers with corporate legitimacy and ensures trust in fiduciary relationships, and therefore the social license to operate.

Keywords: Socio-environmental. Legitimacy. Fiduciary. Stakeholder.

\section{INTRODUÇÃO}

As organizações têm relacionamentos com uma infinidade de stakeholders, cada qual com seus interesses e necessidades. Esses relacionamentos são pautados em dois princípios, sendo eles a Legitimidade Corporativa e o Stakeholder Fiduciário (FREEMAN, 1984). Os princípios de relacionamento com os stakeholders 
foram definidos pela Teoria dos Stakeholders (FREEMAN, 1984), no qual o primeiro constitui-se do dever de os gestores gerenciarem a organização para o benefício de seus stakeholders, inclusive os shareholders, e o segundo constitui-se de uma relação de confiança na qual os gestores devem agir no interesse das partes interessadas, como seu agente, e no interesse da organização, para garantir a sobrevivência dela (EVAN; FREEMAN, 1988). Dos stakeholders que se relacionam com a empresa e que estão mais próximos de suas externalidades está a vizinhança, e num plano já um pouco mais indireto, a sociedade.

Portanto, com base na proposição de que ações sociais empreendidas por um programa socioambiental atendem os dois princípios de relacionamento, este estudo propõe explicar a seguinte questão de partida: Como e por quê um projeto socioambiental contribui no atendimento dos princípios de relacionamento com os stakeholders? A metodologia adotada é de estudo de caso único (YIN, 2010), portanto, qualitativa, com finalidade explicativa, e faz uso da estratégia da proposição teórica (YIN, 2010) para explicar o fenômeno.

Usa-se a técnica da Análise de Conteúdo (BARDIN, 2014) para auxiliar na categorização e organização das informações, bem como o aplicativo Nvivo, um software exclusivo para estudos qualitativos. A empresa objeto deste estudo, a Termotécnica, é líder no setor em que opera, sua matéria-prima é o Poliestireno Expandido - Styrofoam (EPS), conhecido por isopor no Brasil, e o fenômeno a ser analisado compreende a contribuição do Programa Reciclar EPS no atendimento dos princípios de relacionamento com os stakeholders. Este programa faz uso da logística reversa, envolve parcerias em toda a cadeia pós-venda, é de abrangência nacional e já recebeu prêmio nacional de sustentabilidade (OLIVEIRA, 2015a).

Da contribuição dos resultados espera-se proporcionar a compreensão de como e por quê o atendimento dos princípios da Legitimidade Corporativa e Stakeholder Fiduciário são atendidos por meio de um projeto socioambiental, quais são os benefícios para a empresa, para a sociedade e ao meio ambiente.

Este artigo inicia com a apresentação dos princípios de relacionamento com os stakeholders, sendo estes a Legitimidade Corporativa e o Stakeholder Fiduciário (FREEMAN, 1984). Segue com a descrição da empresa que é objeto deste estudo de caso, as características do EPS e do processo produtivo da empresa, descreve o Programa Reciclar EPS, a metodologia de estudo desta pesquisa, os dados da pesquisa, a descrição dos fatos empíricos analisados, a análise e discussão dos Revista Produção Online. Florianópolis, SC, v.17, n. 3, p. 974-997, 2017. 
resultados e a conclusão.

\section{PRINCÍPIOS DE RELACIONAMENTO COM OS STAKEHOLDER - 'LEGITIMIDADE CORPORATIVA’ E 'STAKEHOLDER FIDUCIÁRIO’}

A relação das empresas com seus diversos stakeholders fundamenta-se em dois princípios básicos, Legitimidade Corporativa e Stakeholder Fiduciário (EVAN; FREEMAN, 1988; FRIEDMAN; MILES, 2009). O primeiro princípio constitui-se do dever de os gestores gerenciarem a organização para o benefício de seus stakeholders e o segundo constitui-se de uma relação de confiança na qual os gestores devem agir no interesse das partes interessadas como seu agente (EVAN; FREEMAN, 1988).

O segundo princípio refere-se à fidúcia, que quer dizer confiança (FREEMAN, 1984; HOUAISS; VILLAR, 2011), que deve ser estabelecida entre os gestores e os diversos stakeholders. Esse princípio está relacionado à confiança entre as partes, no qual o trustor, que nesse caso são os stakeholders, depositam nos trustee, que nesse caso são os fideicomissários, ou seja, os gestores da organização, os quais ganham um voto de confiança para operar os ativos (HOUAISS; VILLAR, 2011; SACCONI, 2008).

A relação fiduciária fundamenta-se na confiança e boa-fé que deve existir entre as partes de uma relação (KUBASEK et al., 2009). O fiduciário tem o dever de lealdade, de agir principalmente para aquele que the confiou os assuntos relacionados com a atividade e não para seu próprio interesse pessoal (ATHERTON; BLODGETT; ATHERTON, 2011).

Portanto, a observação desses dois princípios conduz a empresa a adotar um comportamento socialmente responsável, pois essa é uma forma de assegurar a obtenção da legitimidade corporativa e da licença social para operar (SACCONI, 2008).

Por comportamento socialmente responsável entendem-se algumas variáveis estratégicas que são percebidas pelos consumidores e, por conseguinte, afetam seu nível de satisfação, sendo essas variáveis custo, qualidade, tempo, inovação e flexibilidade (PETROVIĆ-RANĐELOVIĆ; STEVANOVIĆ; IVANOVIĆ-ĐUKIĆ, 2015). Também compreende transparência (FLYVERBOM; CHRISTENSEN; HANSEN, 2015), comprometimento e responsabilidade pessoal (TOSTI; HERBST, 2009; Revista Produção Online. Florianópolis, SC, v.17, n. 3, p. 974-997, 2017. 
YOUNG; MAKHIJA, 2014), bem como a adoção de práticas da responsabilidade social e ambiental (TSOI, 2010).

Quanto à licença social para operar, essa se refere à aprovação pelas pessoas afetadas pelos impactos socioambientais na comunidade local ou vizinhança da organização (MELÉ; ARMENGOU, 2016) e decorre de uma percepção de que uma dada ação (ou ações) da empresa é consistente com as normas, padrões, valores e expectativas socialmente aceitas (OLIVER, 1996; SUCHMAN, 1995). Para que a licença social torne-se uma realidade é necessária uma ampla aceitação das atividades da empresa por esse grupo de stakeholders (SLACK, 2008), ou seja, grupo das partes interessadas que não está diretamente envolvido nas transações econômicas com a empresa (MITCHELL; AGE; WOOD, 1997),

Esse grupo de stakeholders compõe-se da comunidade/sociedade, agentes da sociedade civil, agentes reguladores (HENRIQUES; SADORSKY, 1999; KLASSEN; MCLAUGHLIN, 1996; WADDOCK; GRAVES, 1997), grupos de interesse público e grupos profissionais, tais como sindicatos e associações (ETZION, 2007).

Num sentido amplo, a Licença Social para Operar, em inglês Social Licence to Operate (SLO), refere-se a obter aprovação das pessoas afetadas pelas atividades com certo impacto nas comunidades locais ou vizinhança (MELÉ; ARMENGOU, 2016). Essa condição na qual a empresa fica exposta pode influenciar nas decisões e escolhas dos gestores levando-os a criar programas, planos ou ações que venham satisfazer os interesses desses stakeholders (SACCONI, 2008). A SLO se dá quando existe uma ampla aceitação das atividades da empresa pela comunidade local ou sociedade onde essa empresa está a operar (SLACK, 2008). A necessidade da legitimidade social surge porque as empresas operam na sociedade por meio de um contrato social (SHOCKER; SETHI, 1973) e sendo a empresa uma parte integrante dessa sociedade, a sua existência, continuidade e crescimento em grande medida contam com o apoio dessa mesma sociedade (SETHI, 1975; SHOCKER; SETHI, 1973), da legitimidade social para operar (ABERNETHY; WAI FONG, 1996).

A legitimidade corporativa, por sua vez, pode ser compreendida como um modo de os gestores gerenciarem a organização no interesse dos stakeholders (EVAN; FREEMAN, 1988), considerando nesse grupo os shareholders. Essa legitimidade pode ser adquirida a partir de três modos de gerir os negócios, legitimidade moral, pragmática e cultural (SUCHMAN, 1995). A legitimidade moral Revista Produção Online. Florianópolis, SC, v.17, n. 3, p. 974-997, 2017. 
refere-se aos juízos morais conscientes nos produtos, procedimentos, estruturas e líderes da organização (PALAZZO; SCHERER, 2006). Ela é socialmente construída e faz uso da razão para justificar certas ações, práticas ou instituições, entretanto deve estar baseada em profundos princípios éticos (MELÉ: ARMENGOU, 2016). Essa legitimidade reflete uma proatividade social lógica que difere fundamentalmente dos interesses próprios e é resultado dos discursos explícitos publicamente sobre o comprometimento da organização com as questões morais da sociedade (SUCHMAN, 1995).

A prática do comprometimento explícito é uma forma de envolver os gestores e colaboradores na responsabilidade socioambiental da empresa e o uso de códigos e políticas socioambientais contribui para orientar os procedimentos dos gestores (EPSTEIN, 2008).

Legitimidade pragmática, por sua vez, resulta de cálculos de indivíduos cujo interesse é atender as suas próprias vontades (SUCHMAN, 1995). O público pode conferir à empresa a legitimidade pragmática quando detém pensamentos de que a empresa atende seus melhores interesses e/ou partilha seus valores, ou ao atribuirIhe valores de honestidade, confiabilidade e dignidade (SUCHMAN, 1995).

A legitimidade cognitiva, por sua vez, é obtida quando o contexto social refere à empresa e seus produtos, procedimentos, estrutura e comportamento do líder como inevitáveis e necessários. Sua aceitação está baseada em algumas premissas tomadas como certas e amplamente partilhadas (HANNAN; CARROII, 1992). Opera principalmente no nível do subsconsciente, tornando dificultoso para a corporação influenciar diretamente e manipular estrategicamente as percepções (OLIVER, 1991; SUCHMAN, 1995).

Quanto aos stakeholders, estes podem ser divididos em dois grupos, o strict sense, que são aqueles que têm relação mais estrita com a organização, decorrente de algum tipo de investimento nela realizado, e os broad sense, que são aqueles que apenas sofrem os efeitos externos positivos ou negativos das externalidades da organização (SACCONI, 2008).

Os stakeholders strict sense encontram-se numa relação de dependência recíproca, o que faz com que essa relação movimente-se conforme as forças e interesses das partes (SACCONI, 2008). Numa relação de confiança, esses stakeholders acabam por influenciar de forma direta a tomada de decisão dos gestores, afetando o comportamento da empresa (WARBURTON, 2011), uma vez Revista Produção Online. Florianópolis, SC, v.17, n. 3, p. 974-997, 2017. 
que esses mantêm relações diretas e de interesse com a empresa.

Por outro lado, os broad sense não chegam a influenciar diretamente a tomada de decisão, uma vez que não têm relações diretas com a empresa e também não têm interesses nos negócios dela, todavia podem influenciar seu comportamento nas situações em que a relação de confiança não fique clara entre ambos e/ou nas situações em que a empresa demonstre práticas de menos-valia em relação aos valores sociais desses stakeholders. Esse comportamento pode levar à insegurança na relação e, por consequência, afetar a aceitação da sociedade relativamente às práticas industriais da empresa.

Desta forma, nas situações em que aconteça algum impacto socioambiental que afete o bem-estar desse grupo de stakeholders, ou mesmo o seu conjunto de valores, pode esse grupo criar respostas negativas contra a empresa a ponto de afetá-la na sua situação financeira ou nas suas receitas (GODFREY; MERRILL; HANSEN, 2009; JO; NA, 2012; SALAMA; ANDERSON; TOMS, 2011), portanto, podem colocar em risco seus ativos, bem como, sua própria sobrevivência.

Esses princípios de relacionamento podem, portanto, ser interpretados como conduta de comportamento dos gestores, na qual esses devem gerir a empresa no interesse dos stakeholders. Nesse contexto pode ser inserida a RSC, que, vista sob a óptica fiduciária, pode ser compreendida como um modelo estendido de governança corporativa em que aqueles que dirigem a organização têm responsabilidades de cumprimento dos seus deveres fiduciários para com os shareholders e para com os demais stakeholders (SACCONI, 2006).

Ainda nesse cenário de atendimento aos princípios de relacionamento, empresas tendem a divulgar um maior grupo de determinados indicadores em detrimento de outros, opção que pode estar relacionada ao aparato regulatório do país, ou aos relacionamentos com os stakeholders (OLIVEIRA et al., 2009). A divulgação parcial de informações socioambientais deve ser observada com cuidado pelos gestores, uma vez que podem afetar a relação de confiança entre os stakeholders e a empresa, pelo fato de não ficar claro nessa relação o cumprimento do atendimento aos valores da sociedade por completo, mas apenas do que a lei exige.

\section{A EMPRESA E O PROGRAMA RECICLAR EPS}


EPS é a sigla internacional do Poliestireno Expandido, de acordo com a Norma DIN ISSO-1043/78, e, no Brasil, é conhecido como Isopor®, marca registrada da Knauf Isopor Ltda. Foi descoberto em 1949 pelos químicos Fritz Stastny e Karl Buchholz (ABRAPEX, 2016). É um plástico celular rígido, resultante da polimerização do estireno em água, não utilizando o gás CFC ou substitutos, mas o pentano como agente expansor; apresenta inúmeras aplicações em embalagens industriais, artigos de consumo (caixas térmicas, pranchas, porta-gelo etc.), agricultura e construção civil (ABRAPEX, 2016).

O EPS apresenta baixo custo de produção, facilidade de processamento e boas propriedades mecânicas (ABRAPEX, 2016; CANEVAROLO JR., 2010). Seu produto final é o isopor, que é composto de pérolas de até três milímetros de diâmetro, constituindo-se em até $98 \%$ de ar e apenas $2 \%$ de poliestireno. É inerte e inócuo, não contamina o solo, água e ar e não é biodegradável, mas é $100 \%$ reaproveitável e reciclável e pode voltar à condição de matéria-prima (ABRAPEX, 2016; ACEPE, 2016).

Entretanto, o EPS não está totalmente livre de ser considerado poluente, uma vez que suas moléculas ainda são encontradas em águas doces e marinhas, causando poluição junto aos demais plásticos (CONVEY; BARNES; MORTON, 2002; KWON et al., 2015). Foram também encontradas partículas microscópicas de poliestireno expandido dentro de mexilhões (Ml et al., 2016), na composição de microplásticos que contamiman o meio marinho (SONG et al., 2015) e em areias de praias (DAVIS; MARPHY, 2015; KIM et al., 2015). As bolinhas do isopor, quando no meio-ambiente, acabam sendo confundidas com alimentos por peixes e aves aquáticas (FAURE et al., 2015).

Há estudos que apontam para o uso de larvas de Tenebrio Molitor, a larva do bicho-da-farinha, que pode alimentar-se do isopor sem prejudicar sua saúde e que como produto geram o $\mathrm{CO}_{2}$ e excrementos (BBC, 2015; YANG et al., 2015a; 2015b). O uso do Tenebrio Molitor poderia ser adotado nas usinas de reciclagem e um novo produto poderia ser desenvolvido pela empresa.

A logística reversa constitui-se de um conjunto de ações, procedimentos e meios utilizados para viabilizar a coleta e a restituição dos resíduos sólidos ao setor empresarial, para reaproveitamento, em seu ciclo ou em outros ciclos produtivos, ou outra destinação final ambientalmente correta, conforme define a Política Nacional de Resíduos Sólidos (BRASIL, 2010). Pode-se citar o caso da empresa HewlettRevista Produção Online. Florianópolis, SC, v.17, n. 3, p. 974-997, 2017. 
Packard Brasil, que no seu projeto SmartWaste faz uso da tecnologia RFID - Radio Frequency Identification na logística reversa do lixo eletrônico (OLIVEIRA; MARINS; MUNIZ JR., 2016).

Portanto, a partir de uma consciência ecológica e social, a Termotécnica, uma empresa que atua no setor químico, líder no mercado brasileiro de embalagens de produtos e maior transformadora de EPS da América Latina (OLIVEIRA, 2015a), desenvolveu o Programa Reciclar EPS. Esse programa utiliza a logística reversa, é pioneiro no país e já existe há 15 anos (OLIVEIRA, 2015b). A proposta do programa é incorporar o material coletado nos processos industriais, desde a fase de produção da matéria-prima até o produto final, por meio da reciclagem com posterior reintrodução no processo ou no mercado. A reciclagem desse produto acaba por produzir itens de consumo como rodapés, frisos, decks e novas embalagens.

O programa é também uma forma de atender a pressão regulatória (SCOTT, 2001) exercida pelo governo federal por meio da Política Nacional de Resíduos Sólidos, a qual reúne um conjunto de princípios, objetivos, instrumentos, diretrizes, metas e ações com vistas à gestão integrada e ao gerenciamento ambientalmente adequado dos resíduos sólidos (BRASIL, 2010). O Programa Reciclar EPS vem ao encontro do que defendem pesquisadores da área, quando alegam que medidas antipoluidoras apresentam benefícios muito maiores do que seu custo (YANG et al., 2010).

Em 2012 foram geradas aproximadamente 62.730.096 toneladas de resíduos sólidos urbanos no Brasil, do material coletado, 13,5\% cerca de 7.635 .851 toneladas, são materiais plásticos, segundo a Associação Brasileira de Limpeza Pública e Resíduos Especiais - Abrelpe (ABRELPE, 2012). Atualmente, quase um terço, aproximadamente 14 mil toneladas por ano, são reciclados pelo programa (OLIVEIRA, 2015a). Do EPS pós-consumo do tipo embalagens de EPS, apenas $30 \%$ são recicladas e reutilizadas pela Termotécnica (OLIVEIRA, 2015b).

O produto da reciclagem é utilizado na construção civil no sistema construtivo Monoforte, desenvolvido pela própria Termotécnica, o qual consiste no uso de painéis produzidos de EPS e malha de aço (OLIVEIRA, 2015b). É também reutilizado na produção de embalagens novas.

Portanto, com o propósito de melhorar seus índices socioambientais e econômicos, bem como de tornar-se uma empresa cidadã, a empresa adota uma estratégia do tipo ganha-ganha (FALCK; HEBLICH, 2007), na qual todos os Revista Produção Online. Florianópolis, SC, v.17, n. 3, p. 974-997, 2017. 
envolvidos no processo são beneficiados pelo programa. As parcerias realizadas pelo programa envolvem as redes varejistas e cooperativas e a partir dessas estão envolvidos os catadores, que são os indivíduos que efetivamente coletam o material.

\subsection{Estrutura e resultados do Programa Reciclar EPS}

Com a ampla aceitação do programa, a crescente necessidade de atividades socioambientais e a necessidade de que o programa apresente efetividade, a empresa criou uma área especialista para assumir a responsabilidade pelo programa. Essa nova área recebeu o nome de Unidade de Reciclagem - UR. Em termos de estrutura hierárquica, ela é independente e mantém-se com recursos próprios, oriundos do próprio programa. Para dar origem a essa unidade foram investidos $R \$ 15$ milhões, aproximadamente, para efetivamente tornar-se uma atividade industrial e economicamente viável (OLIVEIRA, 2015a). Uma vez criada a UR e verificada a sua realidade de projeto e execução, foi então essa ideia estendida para as outras seis unidades industriais da empresa, localizadas em diferentes cidades do Brasil (OLIVEIRA, 2015a).

Quanto aos resultados do Programa Reciclar EPS, esses proporcionaram à empresa a otimização no uso dos recursos energéticos e redução de $20 \%$ do consumo de estireno e de matéria-prima virgem (TERMOTÉCNICA, 2014). Além desses benefícios internos, o processo de reciclagem e reintrodução do EPS no mercado gerou mais de 100 empregos diretos, conta atualmente com 1.200 pontos de coleta no país, 171 organizações parceiras, 136 gerenciadores de resíduos sólidos e aumento de renda para cinco mil famílias, reunidas em 391 cooperativas (OLIVEIRA, 2015a; TERMOTÉCNICA, 2014). A Figura 1 apresenta o fluxo de movimentação do Programa Reciclar EPS em 2016.

Figura 1 - Configuração do Programa Reciclar EPS - ano 2016 


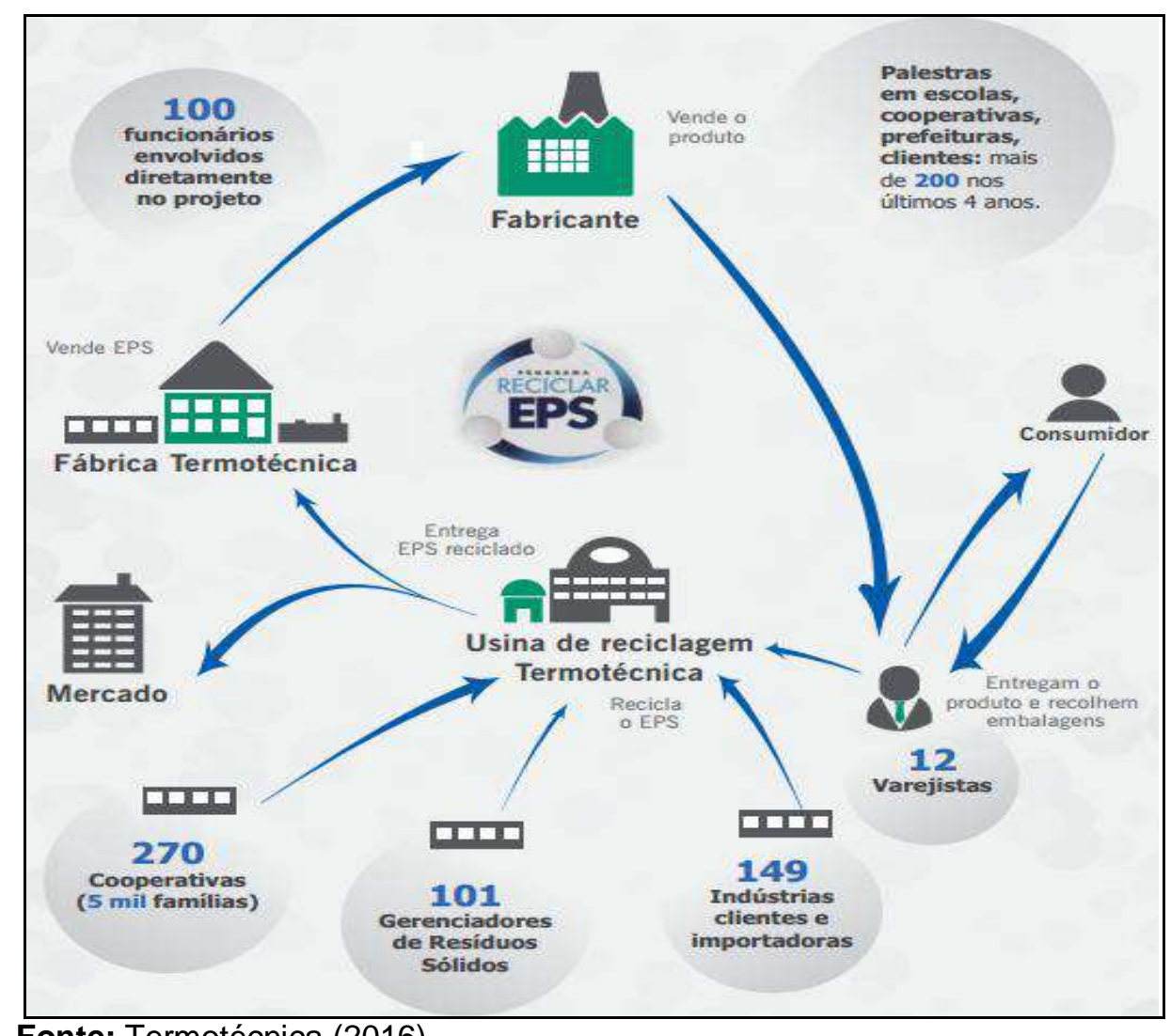

Fonte: Termotécnica (2016)

Por meio da Figura 1 verifica-se a estrutura do programa, no qual são percebidos todos os agentes envolvidos no ciclo, a contar com a própria Termotécnica, o fabricante de EPS, os consumidores, os varejistas, as indústrias clientes e importadores, os gerenciadores de resíduos sólidos, as cooperativas e as cinco mil famílias, bem como o próprio mercado. Estão também envolvidos os funcionários, que nesse caso estão lotados na UR, e a comunidade, por meio de palestras em escolas, prefeituras, cooperativas e clientes.

O programa tem um ciclo que inicia na empresa, passa pelo mercado consumidor e os diversos agentes que dele dependem e finaliza na própria empresa. Nesse ciclo, são beneficiadas de forma direta as famílias de catadores de isopor.

\section{METODOLOGIA}

Este é um estudo qualitativo que adota o estudo de caso único (YIN, 2010) como estratégia de pesquisa. Fez uso do estudo de caso porque o fenômeno acontece na vida real da empresa (YIN, 2010). A estratégia de análise adotada é a proposição teórica (YIN, 2010), construída a partir dos conceitos dos princípios de 
relacionamento com os stakeholders Legitimidade Corporativa e Stakeholder Fiduciário (FREEMAN, 1984). Esses princípios fundamentam-se na afirmação de que os gestores devem gerir a empresa no atendimento de seus stakeholders (FREEMAN, 1984).

Portanto, pressupõe-se que um programa socioambiental de reciclagem, que envolve toda a cadeia pós-venda, retornando os resíduos para a própria empresa, e cujos envolvidos também se beneficiam nesse processo, contribui para atender os dois princípios de relacionamento com os stakeholders.

A considerar que este estudo propõe explicar um fenômeno que acontece no dia a dia das empresas, optou-se por coletar os dados por meio de entrevistas com os envolvidos diretamente com o programa na empresa Termotécnica. Nesse sentido, foram entrevistados o coordenador das áreas ambiental e social, bem como o diretor de Sustentabilidade, por também estar envolvido diretamente com o programa. A entrevista foi do tipo semiestruturada e, para evitar qualquer viés na análise, realizou-se a triangulação das fontes (YIN, 2010), assim, as outras fontes de dados adotadas foram o Relatório de Sustentabilidade, webpage da organização e artigos publicados em revistas.

Para a análise dos dados empíricos, uma vez que este estudo foi construído a partir de uma proposição teórica como estratégia geral de pesquisa, adotou-se a questão de investigação como orientadora da análise, conforme observa Yin (2010). Nesse sentido, a questão geral de pesquisa busca responder: Como e po quê um projeto socioambiental contribui no atendimento dos princípios de relacionamento com os stakeholders?

Adotou-se a técnica da Análise de Conteúdo (BARDIN, 2014) para auxiliar na organização e análise dos dados. De acordo com Bardin (2014), a análise de conteúdo é um "conjunto de técnicas de análise das comunicações" que tem por finalidade a interpretação dessas mesmas comunicações, que podem ser qualquer material verbal ou não verbal, como jornais, revistas, livros, gravações, entrevistas, filmes, entre outros. A análise de conteúdo, para esse autor, é uma metodologia aplicada para aperfeiçoar a interpretação e compreensão do conteúdo de um ou vários documentos que se encontram no estado bruto e precisam ser processados para gerar informações. Segundo Moraes (1999), essa análise ajuda a reinterpretar as mensagens e a atingir uma compreensão de seus significados num nível que vai além de uma leitura comum.

Revista Produção Online. Florianópolis, SC, v.17, n. 3, p. 974-997, 2017. 
Essa é uma técnica utilizada em estudos qualitativos devido à sua característica de categorizar os dados nas categorias ou subcategorias pertinentes, de forma a permitir análise por grupos de informação (BARDIN, 2014). As categorias podem ser definidas a partir de um referencial teórico estabelecido previamente, ou a partir das evidências que os elementos do próprio fenômeno em estudo indicam (BARDIN, 2014). Essas categorias podem ser construídas adotando-se uma hierarquia entre elas, ou como categorias independentes. O tipo hierárquico tem no seu primeiro nível as categorias e, no segundo nível em diante, as subcategorias (BARDIN, 2014).

Para este estudo adotou-se o modelo hierárquico e, para a definição da categoria, adotou-se o referencial teórico previamente estabelecido, portanto, adotou-se Princípios de Relacionamento com os Stakeholders (FREEMAN, 1984) como categoria, por se entender que essa abrange os dois princípios: Legitimidade Corporativa e Stakeholder Fiduciário.

Em seguida foram criadas as subcategorias, dessa vez adotando-se o referencial teórico e os elementos do Programa Reciclar EPS. Nesse sentido, definiram-se as subcategorias Comprometimento com a Responsabilidade Socioambiental, Resultados do Programa, Ações Socialmente Responsáveis e Ações do Tipo Ganha-ganha. Essas três últimas têm origem nos dados da pesquisa. Seguem explicações dessas subcategorias:

a)Comprometimento com a Responsabilidade Socioambiental - Compõe-se de uma declaração expressa publicamente pela organização referente a seu comprometimento com a responsabilidade socioambiental. Esse comprometimento é assegurado com a definição de políticas e códigos sociais e ambientais (SUCHMAN, 1995; Epstein, 2008). Esta subcategoria tem por finalidade verificar se há congruência entre a gestão da empresa ao criar o programa e o seu comprometimento com a própria responsabilidade socioambiental;

b)Resultados do Programa - Correspondem aos resultados das ações do programa. Esta subcategoria tem por finalidade verificar se há alinhamento entre a proposta do programa e os valores socioambientais da empresa;

c) Ações Socialmente Responsáveis - Correspondem às ações realizadas pelo programa. Esta subcategoria tem por finalidade verificar se as ações realizadas atendem às necessidades dos stakeholders;

Revista Produção Online. Florianópolis, SC, v.17, n. 3, p. 974-997, 2017. 
d) Ações do Tipo Ganha-ganha - Correspondem aos efeitos das ações. Tem por finalidade reforçar a subcategoria $\mathrm{c}$ ao analisar os benefícios e beneficiários do programa.

Para auxiliar na análise foi utilizado o aplicativo Nvivo (QSR, 2016), um software específico para pesquisas qualitativas. Esse software adota em sua configuração a mesma técnica da Análise de Conteúdo, ou seja, faz uso de categorias e subcategorias, entretanto neste sistema são denominadas nós e subnós, respectivamente.

A análise dos dados empíricos foi desenvolvida em dois modos, inicialmente descreve-se analiticamente o Programa Reciclar EPS na sua estrutura, resultados e ações. Num segundo momento desenvolve-se a explicação, cuja construção do conhecimento é realizada a partir dos princípios de relacionamento em relação às ações categorizadas nas subcategorias.

Para auxiliar na análise fez-se uso das técnicas Pesquisa de Texto, Frequência de Palavras e Nuvem de Palavras disponibilizadas pelo Nvivo. A primeira técnica faz uma busca nos relatos dos entrevistados, com a finalidade de evidenciar ações em comum e que tenham relevância com o que se busca na pesquisa. A segunda técnica efetua uma busca nos relatos e tem por finalidade identificar as palavras mais repetidas, listando-as em ordem de maior ocorrência. Todavia, para a apresentação dos resultados, pode o autor selecionar as palavras a serem apresentadas. A terceira técnica apresenta as palavras com maior intensidade de uso e sua busca se dá em todos os relatos (QSR, 2016).

\section{DESCRIÇÃO DOS RESULTADOS}

Para compreender se o programa atende os princípios de relacionamento com os stakehoders é necessário verificar a existência de duas informações: a primeira é saber se a empresa se compromete explicita e publicamente com a responsabilidade socioambiental e a segunda é conhecer as necessidades dos stakeholders. Evidenciou-se que a empresa se compromete expressa e publicamente, conforme Relatório de Sustentabilidade: 
preservando o meio ambiente (TERMOTÉCNICA, 2014).

Também foram identificadas as necessidades dos stakeholders, que estão contempladas no documento Matriz de Materialidade, divulgado no Relatório de Sustentabilidade. Portanto, as necessidades identificadas foram as seguintes: Desempenho Econômico; Água, Energia e Ecoeficiência; Emissões, Efluentes e Resíduos; Impacto Ambiental de Atividades, Produtos e Serviços; Saúde e Segurança; Treinamento e Educação; Conduta e Direitos Humanos; e Inovação e Qualidade em Produtos e Serviços (TERMOTÉCNICA, 2014).

Devem também ser levados em consideração os valores da sociedade, os quais na sociedade brasileira envolvem a valorização do meio-ambiente, a valorização das relações sociais, uma característica de ser coletivista, em que o social é mais importante do que o individual, e um forte sentimento de solidariedade (LEMAITRE; HELMSING, 2012; MOTTA, 2003; 2006).

Para a explicação do fenômeno, uma vez que se adotou a técnica da proposição teórica, é inicialmente desenvolvida uma análise analítica do fenômeno, apresentada na forma descritiva. Em seguida é desenvolvida a resposta da questão geral de pesquisa, na forma dissertativa.

Inicialmente apresenta-se a descrição analítica do Programa Reciclar EPS e nessa etapa fez-se uso das três técnicas disponibilizadas pelo Nvivo. A descrição está focada na identificação dos resultados do programa e nas ações do programa. Portanto, na primeira análise, que fez uso da Pesquisa de Texto, os dados analisados evidenciaram que o programa está focado na reciclagem, reutilização do EPS coletado, parcerias e estrutura organizacional. Os benefícios identificados, por sua vez, relacionam-se ao aumento da renda familiar de envolvidos, redução dos resíduos no meio ambiente, redução dos custos ambientais para a empresa, bem como estabelecimento da reputação da empresa como empresa cidadã. Os stakeholders broad sense beneficiados diretamente pelo programa são as famílias de catadores e as lojas varejistas (Quadro 1).

Quadro 1- Resumo das ações, programas e benefícios aos stakeholders 


\begin{tabular}{|c|c|}
\hline AÇÕES & BENEFÍCIOS AOS STAKEHOLDERS \\
\cline { 1 - 2 } Reciclagem & Aumento da renda familiar \\
\cline { 1 - 2 } Reutilização & \multirow{2}{*}{ Redução dos resíduos no meio ambiente } \\
\cline { 1 - 2 } Parcerias & Reputação dos custos ambientais \\
\cline { 1 - 2 } Criação de Unidade de Reciclagem & Rstendido às outras unidades industriais \\
\hline
\end{tabular}

Fonte: Elaborado pelos autores

$\mathrm{Na}$ segunda análise, que fez uso da Frequência de Palavras, os dados confirmam que o projeto está focado na redução de resíduos $(10)(10)$ e na reciclagem do EPS (9)(16), o processo adotado de coleta e restituição de resíduos foi a logística reversa (8)(6) e os benefícios para a empresa foram a redução de custos (10)(6) e asseguração da imagem (2). Para o meio ambiente (4), a redução de impactos ambientais (10)(5)(3), e para a sociedade, trouxe a geração de empregos (4), bem como a geração de renda para as famílias dos catadores. 0 projeto envolve toda a cadeia (4) pós-venda, inserindo-se nessa os catadores (2), por meio das cooperativas (7). É um projeto que apresenta sustentabilidade (5) e ampara-se em parcerias (3), conforme pode ser visualizado no Quadro 2.

Quadro 2 - Programa Reciclar EPS

\begin{tabular}{|c|c|c|c|c|c|}
\hline \multicolumn{7}{|c|}{ Frequência de Palavras - Programa Reciclar EPS } \\
\hline Palavra & Contagem & Palavra & Contagem & Palavra & Contagem \\
\hline eps & 16 & custos & 6 & empregos & 4 \\
\hline redução & 10 & reversa & 6 & ambientais & 3 \\
\hline resíduos & 10 & impactos & 5 & coleta & 3 \\
\hline reciclagem & 9 & sustentabilidade & 5 & parcerias & 3 \\
\hline logística & 8 & ambiente & 4 & catador & 2 \\
\hline cooperativas & 7 & cadeia & 4 & imagem & 2 \\
\hline
\end{tabular}

Fonte: Elaborado pelos autores

A terceira análise, que fez uso da técnica Nuvem de Palavras e é realizada do centro para o exterior da figura, também confirma os dados. Conforme se verifica, palavras como resíduos, redução e EPS, que estão no centro, estão a indicar as ações de maior intensidade do programa. Num segundo plano estão as palavras reciclagem, cooperativas e logística. Esses dois conjuntos de palavras apresentam a 
essência do projeto, que trata de busca da redução dos impactos gerados no meio ambiente por conta da geração de resíduos de EPS. O processo é possível porque fez uso da logística reversa e há envolvimento de cooperativas em toda a cadeia pós-venda como principais parceiras no projeto.

Nas extremidades encontram-se as palavras sustentabilidade, embalagens, impactos, empregos, ambiente, recursos e renda, que podem indicar a natureza sustentável do programa, a geração de riqueza para os stakeholders externos por meio da criação de empregos e aumento de renda familiar (Figura 2).

Figura 2 - Nuvem de Palavras - Programa Reciclar EPS

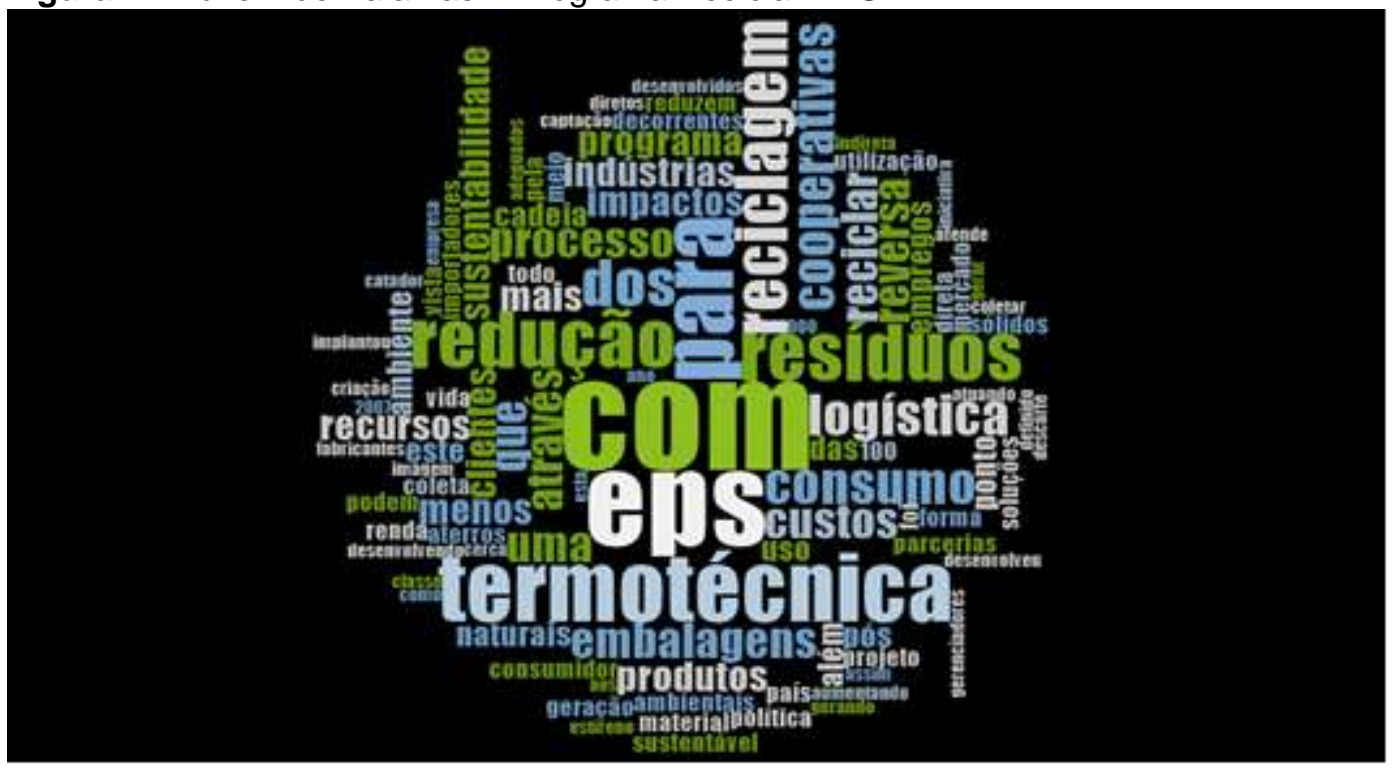

Fonte: Elaborado pelos autores

\section{ANÁLISE E DISCUSSÃO DOS RESULTADOS}

Com base nos dados da pesquisa, confirma-se o pressuposto de que um Revista Produção Online. Florianópolis, SC, v.17, n. 3, p. 974-997, 2017. 
programa socioambiental atende os princípios de relacionamento com os stakeholders. Para a Legitimidade Corporativa, a realização do Programa Reciclar EPS demonstra práticas de uma gestão do tipo "moral" na busca pela legitimidade corporativa (SUCHMAN, 1995) por parte dos gestores da Termotécnica, uma vez que há declaração expressa publicamente do comprometimento da empresa com a responsabilidade socioambiental e houve um comportamento dos gestores em direção ao atendimento dessa responsabilidade quando tornaram realidade 0 Programa Reciclar EPS.

Esse princípio é atendido pelo programa porque seus resultados estão refletidos na redução dos custos de produção, na criação de novos produtos, na imagem de empresa cidadã e pela proteção dos ativos, ao reduzir a exposição da empresa aos riscos ambientais, o que está assegurado pela redução dos impactos ambientais por meio da redução dos resíduos de EPS no meio ambiente. Portanto, o programa atende aos interesses dos stakeholders (EVAN; FREEMAN, 1988), uma vez que assegura a sobrevivência da empresa (EVAN; FREEMAN, 1988), bem como atende as necessidades de eficiência ambiental e de redução dos impactos ambientais previstos na Matriz de Materialidade.

Os gestores do programa ampliaram a logística e a reciclagem, estenderamna para as demais unidades industriais localizadas em outras cidades do país, proporcionaram redução de custo da matéria-prima pela adição do EPS coletado, geraram novos produtos a partir do material coletado e mais 100 novos empregos.

As ações sociais realizadas pelo programa, e nessas incluem-se as do tipo ganha-ganha, também contribuíram para a busca da legitimidade corporativa pelos gestores, uma vez que ao atenderem as necessidades dos stakeholders, em especial os broad sense, evidenciaram por parte desses uma aceitação em relação à gestão da organização, fator que conduz à concessão de uma licença social para operar (MELÉ; ARMENGOU, 2016; SLACK, 2008). Portanto, com essas ações estão os gestores protegendo os ativos da empresa de problemas socioambientais pela obtenção de uma legitimidade social para operar (ABERNETHY; WAI FONG, 1996).

Quanto ao atendimento do princípio Stakeholder Fiduciário, a relação de fidúcia acaba também sendo assegurada pela confiança que fica estabelecida entre as partes (HOUAISS; VILLAR, 2011; SACCONI, 2008), uma vez que a gestão da Termotécnica demonstra publicamente sua preocupação e comprometimento com a sua responsabilidade socioambiental, bem como confirma esse comportamento por Revista Produção Online. Florianópolis, SC, v.17, n. 3, p. 974-997, 2017. 
meio da criação do Programa Reciclar EPS.

A redução de resíduos no meio ambiente e a consequente diminuição dos impactos ambientais proporcionados pelo programa trazem benefícios para a sociedade, podendo-se citar a menor exposição da sociedade aos acidentes ambientais, bem como a possibilidade de viver em um meio-ambiente saudável. Os benefícios desse programa podem ser sentidos pela atual geração, bem como poderão ser sentidos pela geração futura, uma vez que o meio-ambiente encontrarse-á livre dos resíduos de EPS.

Quanto às ações do tipo ganha-ganha, estas também contribuem para atender a relação de fidúcia entre a empresa e os stakeholders broad sense, uma vez que esses foram beneficiados por meio do aumento da renda familiar e da melhoria da qualidade do meio-ambiente. Nesse movimento, a empresa também foi beneficiada, tanto pela redução dos custos industriais quanto pela asseguração da imagem de empresa cidadã.

O projeto atende os dois princípios de relacionamento, o primeiro pelo atendimento pelos gestores ao comprometimento da empresa com a responsabilidade socioambiental, com o resultado econômico, com a proteção dos ativos, e nesses inclui-se a reputação da empresa. $O$ segundo, pelo uso ambientalmente correto dos ativos ambientais fiduciariamente cedidos aos gestores para utilização pela empresa, pela proteção do meio ambiente e pelos benefícios diretos proporcionados aos agentes envolvidos no ciclo do programa.

Uma síntese do exposto, desenvolvida a partir dos dados da análise descritiva, pode ser visualizada no Quadro 03, no qual são evidenciadas as ações com as respectivas categorias.

Quadro 3 - Síntese do atendimento dos princípios de relacionamento com os stakeholders

\begin{tabular}{|c|c|}
\hline Princípios de Relacionamento com os Stakeholders (Legitimidade Corporativa e Stakeholder Fiduciário) \\
\hline Categorias e subcategorias & Evidências \\
\hline Comprometimento & Expresso publicamente no Relatório de Sustentabilidade; Realização do Programa \\
Reciclar EPS.
\end{tabular}

Fonte: Elaborado pelos autores 
Um projeto de reciclagem traz em sua essência uma concepção de redução de custos, reaproveitamento de materiais e redução dos impactos ambientais gerados, por conseguinte, seus efeitos podem ser percebidos nos resultados econômicos da empresa. Todavia, quando um projeto inclui na sua essência a preocupação com a responsabilidade social, está também protegendo a sociedade.

Por meio desse projeto, os stakeholders broad sense são beneficiados porque aumentam sua renda familiar e a sociedade por poder viver num ambiente limpo: $A$ empresa também se beneficia ao diminuir a exposição de seus ativos aos riscos socioambientais, ao manter sua reputação de empresa socialmente responsável e ao assegurar sua sobrevivência no ambiente em que opera.

Novos estudos podem ser realizados a partir desta pesquisa, em especial nas áreas da gestão de resíduos e na responsabilidade social corporativa, em projetos que utilizem a logística reversa ou em pesquisas que tenham por intenção fazer uso do "bicho-da-farinha". Podem também ser desenvolvidos estudos sobre modelos de gestão de projetos socioambientais, estudos que envolvam a imagem da empresa, ou mesmo que aprofundem as pesquisas sobre o atendimento dos princípios de relacionamento com os stakeholders.

\section{CONCLUSÃO E CONSIDERAÇÕES FINAIS}

O presente estudo buscou responder de que forma um projeto socioambiental de reciclagem contribui para atender os princípios de relacionamento com os stakeholders e as respostas obtidas indicam que há um encontro entre as ações dos gestores, os interesses da empresa e dos stakeholders, em especial os broad sense. O método do estudo de caso permitiu conhecer em profundidade o fenômeno, porque alguns dos dados empíricos coletados pertencem ao dia a dia da empresa e não são divulgados nos relatórios externos. A técnica da entrevista semiestruturada permitiu obter detalhes internos do programa e, com isso, perceber o comportamento do programa com os valores da organização, e o uso da webpage e do Relatório de Sustentabilidade como fontes de informações complementares contribuiu na triangularização dos dados e, com isso, para se evitar o viés na análise.

A técnica da Análise de Conteúdo permitiu construir a relação entre os elementos empíricos e, portanto, responder à questão de pesquisa. O método do Revista Produção Online. Florianópolis, SC, v.17, n. 3, p. 974-997, 2017. 
estudo de caso apresenta a vantagem do aprofundamento da análise sobre o fenômeno, porém não permite sua generalização por apresentar a subjetividade como um elemento nas análises (YIN, 2010). Todavia, o uso da técnica da Análise de Conteúdo procura reduzir a subjetividade por meio da eliminação da aleatoriedade da escolha dos elementos da relação, ao categorizar os dados com base no referencial teórico e/ou nas evidências do fenômeno (BARDIN, 2014).

Outro fator que contribui para a certeza das informações é a triangularização das fontes, o que foi feito usando-se dados das entrevistas, webpage, revistas e Relatório de Sustentabilidade. Pontos negativos que possam prejudicar os resultados desta pesquisa não foram observados porque a empresa entrevistada disponibilizou todas as informações que Ihe foram solicitadas, bem como foi feita a triangulação de fontes. Portanto, os resultados desta pesquisa podem contribuir para novos estudos científicos.

\section{AGRADECIMENTOS}

Os autores agradecem à empresa TERMOTÉCNICA, em especial à Regina Zimmermann e Thiago $\mathrm{H}$. dos Santos, pela colaboração para a realização desta pesquisa.

\section{REFERÊNCIAS}

ABERNETHY, M. A.; WAI FONG, C. A Field Study of Control System 'Redesign': The Impact of Institutional Processes on Strategic Choice. Contemporary Accounting Research, v. 13, n. 2, p. 569-606, 1996. doi: 10.1111/j.1911-3846.1996.tb00515.x

ABRAPEX. O que é EPS. São Paulo, 2016. Disponível em:

< http://www.abrapex.com.br/01OqueeEPS.html $>$. Acesso em: 22 jan. 2016

ACEPE. Factos sobre o EPS e o meio ambiente. Lisboa, 2016. Disponível em: < http://www.acepe.pt/index.php/sustentabilidade/o-meio-ambiente/factos-sobre-o-eps-e-omeio-ambiente $>$. Acesso em: 22 jan. 2016.

ATHERTON, S. C.; BLODGETT, M. S.; ATHERTON, C. A. Fiduciary principles: corporate Responsibilities to Stakeholders. Journal of Religion \& Business Ethics, v. 2, n. 2, p. 1-15, 2011.

BARDIN, L. Análise de conteúdo. Lisboa: Edições 70, 2014.

BBC. A larva que come plástico e pode ter papel-chave em reciclagem. Brasil, 2015. Disponível em: <

Revista Produção Online. Florianópolis, SC, v.17, n. 3, p. 974-997, 2017. 
http://www.bbc.com/portuguese/noticias/2015/10/151026 larva poluicao lab >. Acesso em: 22 jan. 2016.

BRASIL. Política nacional de resíduos sólidos. Lei ํㅜ 12.305, de 2 de agosto de 2010. Brasília, 2010.

CANEVAROLO JR, S. V. Ciência dos polímeros: um texto básico para tecnólogos e engenheiros. 3a. São Paulo: Artliber, 2010.

CONVEY, P.; BARNES, D. K. A.; MORTON, A. Debris accumulation on oceanic island shores of the Scotia Arc, Antarctica. Polar Biology, v. 25, n. 8, p. 612-617,2002. doi:10.1007/s00300-002-0391-x

EPSTEIN, M. J. Making sustainability work: best pratices in managing and measuring corporate social, environmental and economic impacts. Sheffield: Greenleaf Publishing, 2008.

ETZION, D. Research on organizations and the natural environment, 1992-Present: A Review. Journal of Management, v. 33, n.4, 2007.

https://doi.org/10.1177/0149206307302553

EVAN, W. M.; FREEMAN, R. E. A stakeholder theory of the modern corporation: Kantian capitalism. Englewwod-Cliff: Prentice Hall, 1988.

FALCK, O.; HEBLICH, S. Corporate social responsibility: Doing well by doing good.

Business Horizons, 50, p. 247-254, 2007. https://doi.org/10.1016/i.bushor.2006.12.002

FAURE, F. et al. Plastic pollution in Swiss surface waters: nature and concentrations, interaction with pollutants. Environmental Chemistry, v. 12, n. 5, p. 582-591, 2015. https://doi.org/10.1071/EN14218

FLYVERBOM, M.; CHRISTENSEN, L. T.; HANSEN, H. K. The Transparency-Power Nexus: Observational and Regularizing Control. Management Communication Quarterly, v. 29, n. 3, p. 385-410, 2015. https://doi.org/10.1177/0893318915593116

FREEMAN, R. E. Strategic management: a stakeholder approach. Boston: Pitman, 1984.

FRIEDMAN, Â. L.; MILES, S. Stakeholders: theory and practice. Oxford: Oxford University Press, 2009.

GODFREY, P.; MERRILL, C. B.; HANSEN, J. M. The Relationship Between Corporate Social Responsibility and Shareholder Value: and Empirical Test of the Risk Management Hipotesis. Strategic Management Journal, 30, p.425-445, 2009. doi:10.1002/smj.750.

HANNAN, M. T.; CARROLL, G. R. Dynamics of organizational populations: density, legitimation and competition. Oxford: Oxford University Press, 1992.

HENRIQUES, I.; SADORSKY, P. THE RELATIONSHIP BETWEEN ENVIRONMENTAL COMMITMENT AND MANAGERIAL PERCEPTIONS OF STAKEHOLDER IMPORTANCE. Academy of Management Journal, v. 42, n. 1, p. 87-99, 1999.

https://doi.org/10.2307/256876

HOUAISS, A.; VILLAR, M. S. Houaiss: dicionário do português atual. LEITORES, C. D.: (s.i.) 2011. 
JO, H.; NA, H. Does CSR Reduce Firm Risk? Evidence from Controversial Industry Sectors. Journal of Business Ethics, 110, p. 441-456, 2012.

KLASSEN, R. D.; MCLAUGHLIN, C. P. The impact of environmental management on firm performance. Management Science, v. 42, n. 8, p. 1199-1214, 1996.

https://doi.org/10.1287/mnsc.42.8.1199

KUBASEK, N. et al. Dynamic business law. New York: McGraw-Hill, 2009.

KWON, B. G. et al. Global styrene oligomers monitoring as new chemical contamination from polystyrene plastic marine pollution. Journal of Hazardous Materials, v. 300, p. 359367, 2015. http://dx.doi.org/10.1016/j.jhazmat.2015.07.039

LEMAITRE, A.; HELMSING, A. H. J. Solidarity Economy in Brazil: Movement, Discourse and Practice Analysis through a Polanyian understanding of the Economy. Journal of International Development, v. 24, n. 6, p. 745-762, 2012. https://doi.org/10.1002/jid.2865

MELÉ, D.; ARMENGOU, J. Moral Legitimacy in Controversial Projects and Its Relationship with Social License to Operate: A Case Study. Journal of Business Ethics, 136, 4, p. 729-742, 2016. https://doi.org/10.1007/s10551-015-2866-z

MI, J. et al. Styrofoam Debris as a Source of Hazardous Additives for Marine Organisms. Environmental science \& technology, v. 50, n.10, p. 4951-60, 2016.

https://doi.org/10.1021/acs.est.5b05485

MITCHELL, R. K.; AGE, B. R.; WOOD, D. J. Toward a Theory of Stakeholder Identification and salience: defining the principle of who and what reality counts. Academy of

Management Review. 22: 853-886 p. 1997.

https://doi.org/10.5465/AMR.1997.9711022105

OLIVEIRA, M. Tudo pelo isopor. Exame. São Paulo: Editora Abril. 1101: 211 p. 2015a. $2015 b$.

Uma opção para levantar paredes. Exame. São Paulo: Editora Abril. 1101: 178 p.

OLIVEIRA, M. C. et al. Disclosure of social information by Brazilian companies according to United Nations indicators of corporate social responsibility. Revista Contabilidade \&

Finanças, 20, p.116-132, 2009.

OLIVEIRA, U. R. D.; MARINS, F. A. S.; MUNIZ JR, J. Logística Reversa e Identificação de Produtos: revisão teórica para indústria eletroeletrônica. Revista Produção Online, v.16, n. 2, p.633-677, 2016. http://dx.doi.org/10.14488/1676-1901.v16i2.2049

OLIVER, C. Strategic Responses to Institutional Processes. Academy of Management Review, v. 16, n. 1, p. 145-179, 1991. https://doi.org/10.5465/AMR.1991.4279002.

OLIVER, C. The Institutional Embeddedness of Economid Activity. Advances in Strategic Management, v.13, sn, p. 163-186, 1996.

PALAZZO, G.; SCHERER, A. G. Corporate Legitimacy as Deliberation: A Communicative Framework. Journal of Business Ethics, v. 66, n. 1, p. 71-88, 2006.

https://doi.org/10.1007/s10551-006-9044-2 
PETROVIĆ-RANĐELOVIĆ, M.; STEVANOVIĆ, T.; IVANOVIĆ-ĐUKIĆ, M. Impact of Corporate Social Responsibility on the Competitiveness of Multinational Corporations.

Procedia Economics and Finance, v. 19, sn., p. 332-341, 2015. http://dx.doi.org/10.1016/S2212-5671(15)00034-9

QSR. (2016). NVivo Getting Started. Disponível em: www.qsrinternational.com.

SACCONI, L. A Social Contract Account for CSR as an Extended Model of Corporate Governance (I): Rational Bargaining and Justification. Journal of Business Ethics, v. 68, n. 3, p. 259-281, 2006. https://doi.org/10.1007/s10551-006-9014-8

SACCONI, L. CSR as Contractarian Model of Multi-Stakeholder Corporate Governance and the Game-Theory of its Implementation. University Of Trento, 2008.

http://dx.doi.org/10.2139/ssrn.1163704

SALAMA, A.; ANDERSON, K.;TOMS, J. S. Does community and environmental responsibility affect firm risk: Evidence from UK panel data 1994-2006. Business Ethics: A European Review, v. 20, n. 2, p. 192-204, 2011. https://doi.org/10.1111/i.14678608.2011.01617.x

SCOTT, R. W. Institutions and Organizations (2nd ed.). Thousand Oaks (CA): Sage Publication, 2001

SETHI, S. P. Dimensions of Corporate Social Performance: An Analytical Framework. California Management Review, v. 17, n. 3, p. 58-64, 1975. Doi: https://doi.org/10.2307/41162149

SHOCKER, A. D., \& Sethi, S. P. An approach to incorporating societal preferences in developing corporate action strategies. California Management Review, v. 15, n. 4, 97-105, 1973.

SLACK, K. Corporate Social License and Community Consent. Policy Innovation, v. 21, sn., p...., 2008. Disponível em:

https://www.carnegiecouncil.org/publications/archive/policy innovations/commentary/000094 l:pf printable/Template=print?version=1470854961

SUCHMAN, M. C. Managing Legitimacy: Strategic and Institutional Approaches. Academy of Management Review, v. 20, n. 3, p. 571-610, 1995.

Termotécnica. Relatório de Sustentabilidade 2014, ano 2014 Disponível em: http://www.termotecnica.ind.br/wp-content/uploads/2015/07/Relatorio Termotecnica.pdf

Termotécnica. Reciclagem. 2016. Disponível em: http://www.reciclareps.com.br/reciclagem/

TOSTI, D.; HERBST, S. A. Organizational Performance and Customer Value. Journal of Organizational Behavior Management, v. 29, n. 3-4, p. 294-314, 2009. doi: https://doi.org/10.1080/01608060903092151

TSOI, J. Stakeholders' Perceptions and Future Scenarios to Improve Corporate Social Responsibility in Hong Kong and Mainland China. Journal of Business Ethics, v. 91, n. 3, p. 391-404, 2010. https://doi.org/10.1007/s10551-009-0091-3

WADDOCK, S. A.; GRAVES, S. B. Finding the Link Between Stakeholder Relations and Quality of Management. The Journal of Investing, v. 6, n. 4, p. 20-24, 1997. doi: 
https://doi.org/10.3905/joi.1997.408435.

WARBURTON, A. J. Do fiduciary duties matter? Corporate Governance: The international journal of business in society, v. 11, n. 5, p. 541-548, 2011. https://doi.org/10.1108/14720701111176957

YANG, Y.; YANG, J.; WU, W. M.; ZHAO, J.; SONG, Y. L.; GAO, L. C.; . . JIANG, L. Biodegradation and Mineralization of Polystyrene by Plastic-Eating Mealworms: Part 1. Chemical and Physical Characterization and Isotopic Tests. Environmental Science \& Technology, v. 49, n. 20, p. 12080-12086, 2015(a). https://doi.org/10.1021/acs.est.5b02661

YANG, Y.; YANG, J.; WU, W. M.; ZHAO, J.; SONG, Y. L.; GAO, L. C.; . . JIANG, L. Biodegradation and Mineralization of Polystyrene by Plastic-Eating Mealworms: Part 2. Role of Gut Microorganisms. Environmental Science \& Technology, v. 49, n. 20, p. 1208712093, 2015(b). https://doi.org/10.1021/acs.est.5b02663

IN, R. K. (2010). Estudo de caso: planejamento e métodos. 4. ed. Porto Alegre: Bookman.

YOUNG, S. L.; MAKHIJA, M. V. Firms' corporate social responsibility behavior: An integration of institutional and profit maximization approaches. Journal of International Business Studies, v. 45, n. 6, p. 670-698, 2014. https://doi.org/10.1057/jibs.2014.29

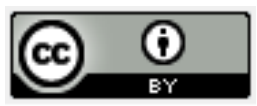

Artigo recebido em 23/02/2017 e aceito para publicação em 11/08/2017 DOI: http://dx.doi.org/10.14488/1676-1901.v17i3.2742 